\title{
Recent Developments in Ni-Mn-Ga Foam Research
}

\section{Peter Müllner, ${ }^{1, a}$, Xuexi Zhang ${ }^{2,3, b}$, Yuttanant Boonyongmaneerat ${ }^{2,4, c}$ Cassie Witherspoon $^{1, d}$, Markus Chmielus ${ }^{1,5, e}$, David C. Dunand ${ }^{2, f}$}

\author{
${ }^{1}$ Department of Materials Science and Engineering, Boise State University, Boise, ID 83725, USA \\ ${ }^{2}$ Department of Materials Science and Engineering, Northwestern University, Evanston, IL 60208, \\ USA \\ ${ }^{3}$ also at Harbin Institute of Technology, West Dazhi Street No. 92, 150001, Harbin, P. R. China \\ ${ }^{4}$ present address: Metallurgy and Materials Science Research Institute, Chulalongkorn University, \\ Pathumwan, Bangkok 10330, Thailand \\ ${ }^{5}$ also at Helmholtz Center for Materials and Energy, Glienicker Str. 100, 14109 Berlin, Germany

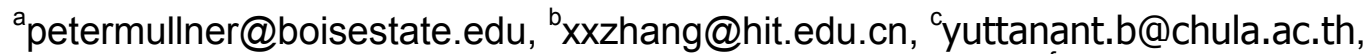 \\ dcassiebassett@u.boisestate.edu, e'markuschmielus@boisestate.edu, ${ }^{f}$ dunand@northwestern.edu
}

Keywords: foam, large strain, polycrystalline, cast structure, fatigue.

\begin{abstract}
Grain boundaries hinder twin boundary motion in magnetic shape-memory alloys and suppress magnetic-field-induced deformation in randomly textured polycrystalline material. The quest for high-quality single crystals and the associated costs are a major barrier for the commercialization of magnetic shape-memory alloys. Adding porosity to polycrystalline magneticshape memory alloys presents solutions for (i) the elimination of grain boundaries via the separation of neighboring grains by pores, and (ii) the reduction of production cost via replacing the directional solidification crystal growth process by conventional casting. Ni-Mn-Ga foams were produced with varying pore architecture and pore fractions. Thermo-magnetic training procedures were applied to improve magnetic-field-induced strain. The cyclic strain was measured in-situ while the sample was heated and cooled through the martensitic transformation. The magnetic field-induced strain amounts to several percent in the martensite phase, decreases continuously during the transformation upon heating, and vanishes in the austenite phase. Upon cooling, cyclic strain appears below the martensite start temperature and reaches a value larger than the initial strain in the martensite phase, thereby confirming a training effect. For Ni-Mn-Ga single crystals, external constraints imposed by gripping the crystal limit lifetime and/or magnetic-field-induced deformation. These constraints are relaxed for foams.
\end{abstract}

\section{Introduction}

The very high magnetic-field-induced strain (MFIS, up to $10 \%$ ) displayed by bulk monocrystalline Ni-Mn-Ga alloys [1-3] is a true plastic strain produced by twin-boundary motion. This strain can be recovered by reverse twin-boundary motion through reorientation of the applied magnetic field and alternatively by mechanical compression perpendicular to the magnetic field direction. Fully recoverable MFIS over $>10^{8}$ magneto-mechanical cycles was reported for monocrystalline bulk $\mathrm{Ni}-\mathrm{Mn}-\mathrm{Ga}$ [4], with very high actuation speed in the $\mathrm{kHz}$ regime, ultimately limited by eddy currents and inertia [5]. The above properties make magnetic shape-memory alloys exceptionally well-suited for applications such as fast actuators with long stroke and high precision (e.g. for engine valves and ultra fast high precision scanners and printers).

A severe shortcoming of magnetic shape-memory alloys is that very large MFIS have, to date, only been achieved for single crystals. Due to constraints imposed by grain boundaries, the MFIS is near zero in randomly textured, fine-grained, polycrystalline $\mathrm{Ni}-\mathrm{Mn}-\mathrm{Ga}$ [6]. To reduce these constraints and increase MFIS, coarse-grained, highly-textured, polycrystalline Ni-Mn-Ga was produced by directional solidification and annealing [7]. These materials displayed a MFIS recovery 
of $1 \%$ after mechanical training [8], and a similar strain when magnetic actuation was combined with acoustic excitation [9].

Magneto-mechanical fatigue of Ni-Mn-Ga single crystals presents another challenge [4, 10-12]. Constraints imposed by gripping the sample cause stresses. The gripping stresses trigger the formation of coarse intersecting twins. Cracks nucleate where these twins intersect [11,13] and grow along twin boundaries during ongoing cyclic loading, ultimately leading to failure $[4,11,12]$.

We have shown that introducing pores in polycrystalline Ni-Mn-Ga reduces the internal constraints of grain boundaries to the point that the material displayed a repeatable MFIS of $0.12 \%$ [14]. By modifying the architecture of foam with a bimodal pore size distribution, the MFIS was recently increased to $8.7 \%$ [15]. The aim of the present paper is to briefly review these recent developments and to discuss the role of porosity in reducing the effects of internal and external constraints on MFIS and magneto-mechanical fatigue.

\section{Experimental}

The processing of Ni-Mn-Ga foam via the replication method was described in Ref. [14]. This method makes use of $\mathrm{NaAlO}_{2}$ powders as space-holder[16], which is pre-sintered to form an openpore preform into which the Ni-Mn-Ga alloy is cast. Different foam architectures can be achieved through variation of the size and shape of the ceramic powder. Foams with two distinct architectures were produced here. For foam I, a $\mathrm{NaAlO}_{2}$ powder with monomodal size distribution between 355 and $500 \mu \mathrm{m}$ was used, leading to a similar pore size distribution of the metallic foam [14]. Foam I with $0.12 \%$ MFIS was cast from an alloy with composition $\mathrm{Ni}_{50.6} \mathrm{Mn}_{28} \mathrm{Ga}_{21.4}$ (numbers indicate atomic percent). For foam II with $8.7 \% \mathrm{MFIS}$, a $\mathrm{NaAlO}_{2}$ powder with bimodal size distribution was used [15], which was blended from two $\mathrm{NaAlO}_{2}$ powders with size 500-600 $\mu \mathrm{m}$ and 75-90 $\mu \mathrm{m}$. Foam II was cast from an alloy with composition $\mathrm{Ni}_{52.2} \mathrm{Mn}_{24.1} \mathrm{Ga}_{23.7}$. Both foams are fully martensitic at room temperature. Fig. 1 shows scanning electron microscopy images of both foams. Fig. 2 shows the twin structures of thick and thin struts. In foam I (with monomodal pore size distribution), some struts have twins that span from one pore to the next. Some thick struts and nodes, however, have multivariant twin structures and even grain boundaries (Fig. 2a). In the thinner struts of foam II (with bimodal grain size distribution), twins span across entire struts from one pore to the next (Fig. 2b).

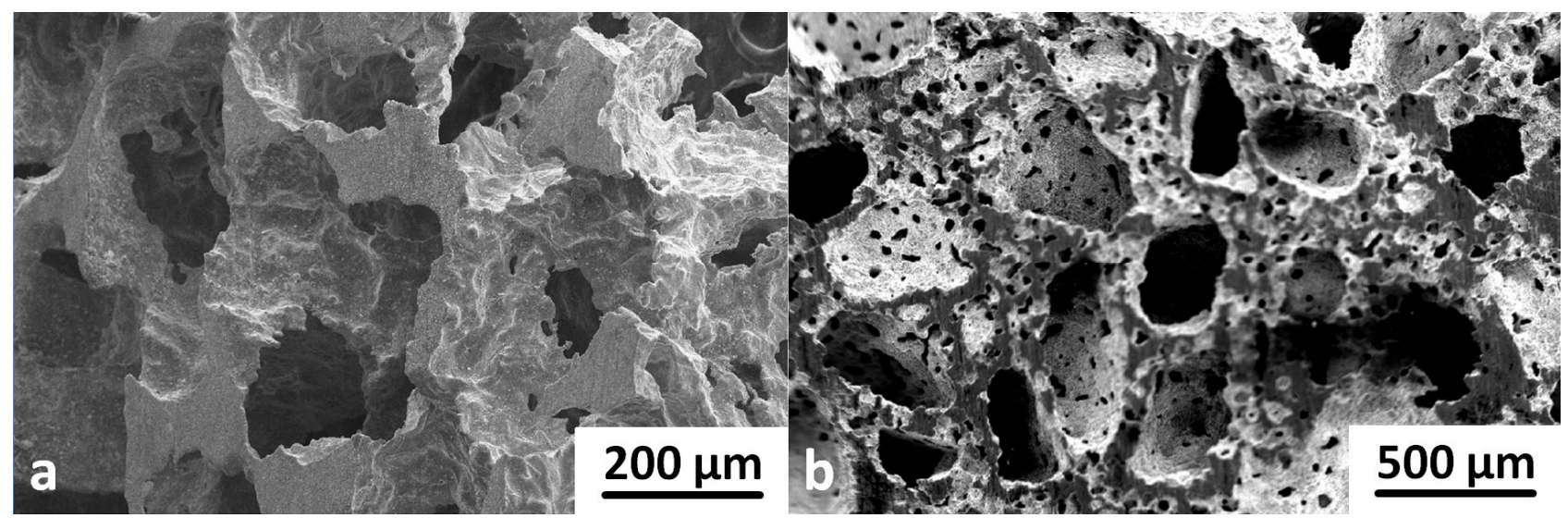

Fig. 1: SEM images of pore structures of (a) foam I with monomodal pores and (b) foam II with bimodal pores 


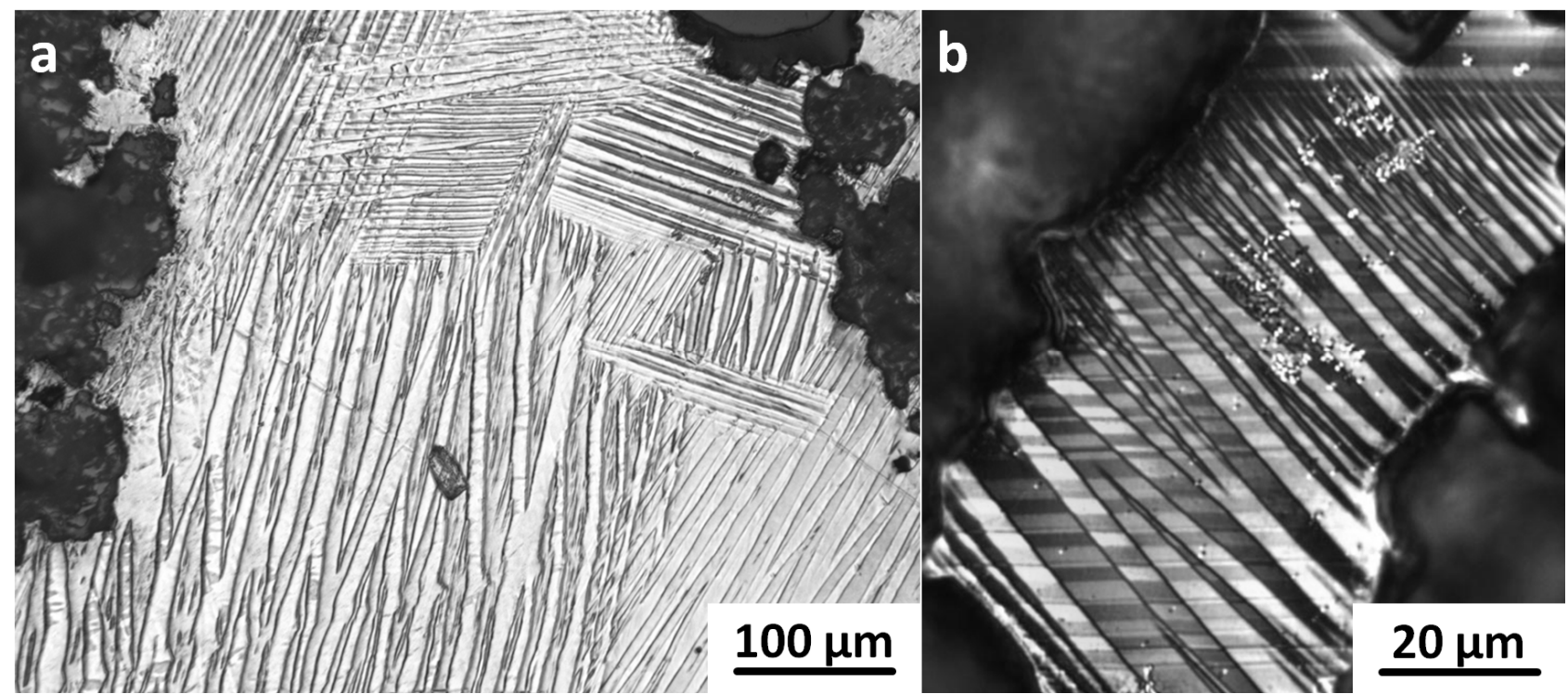

Fig. 2: Twin structure in foam struts. (a) In thick struts such as in foam I, twins do not always span from one pore across the entire strut to the next pore but they form complex arrangements. (b) In thin twins of foam II, most all twins span from one pore to the next. Dark areas are pores.

Magneto-mechanical experiments with a rotating magnetic field were introduced in 2000 [17] and applied for fatigue experiments, first with a slowly rotating field of $2 \mathrm{~T}$ [18] and later in 2004 with a field of $0.97 \mathrm{~T}$ rotating at up to 12,000 revolutions per minute [3] allowing for studying magneto-mechanical fatigue over hundreds of millions of magneto-mechanical cycles [4]. The experimental set-up, which is described in detail in [3] was recently upgraded with heating and cooling capabilities as illustrated in Fig. 3 and described in greater detail in [15]. This new setup enables the detection of MFIS in a rotating magnetic field of $0.97 \mathrm{~T}$ while the sample is heated and cooled through the martensitic transformation.

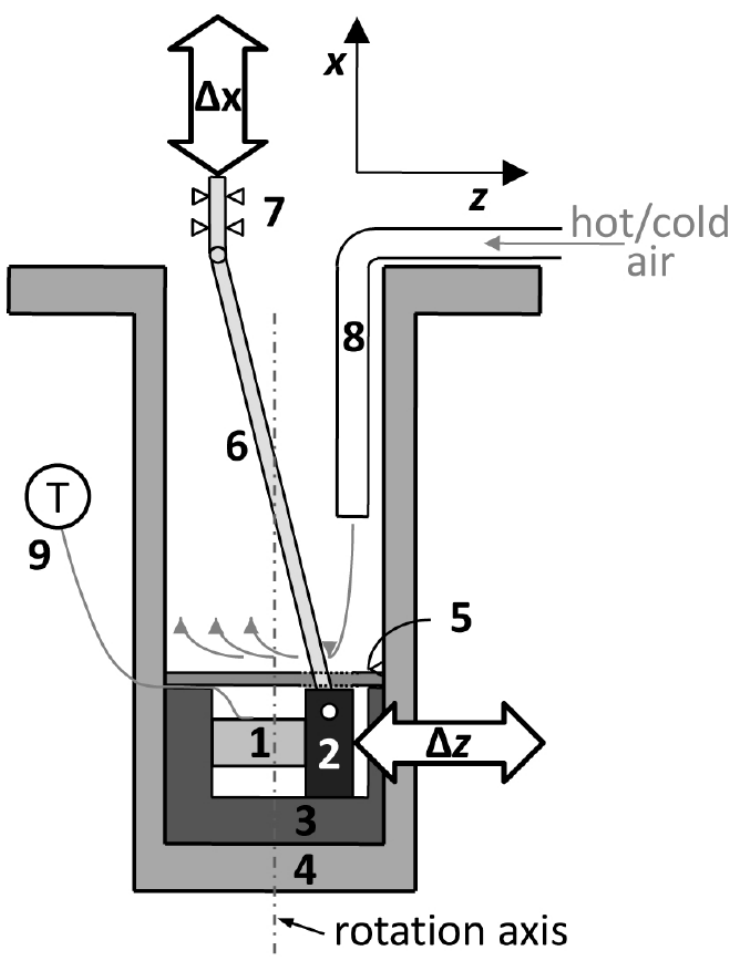

Fig. 3: Schematic of the magneto-mechanical experiment. The foamed sample (1) is glued to the sliding head (2) and holder (3), bolted to a tube (4), which is placed in the rotating field (field rotation axis shown). A lid (5) encloses the foam. A ceramic pushing rod (6) and a redirection mechanism (7) measure the displacement of the foam in its $z$ direction by transforming it to displacement in the $x$ direction which is measured outside the magnetic field with a Heidenhain extensometer type MT1281. A tube (8) is used to direct hot and cold air onto the lid. A thermocouple (9) measures the temperature on the foam surface. The vertical dashed line is the rotation axis of the magnetic field whose vector is oriented perpendicular to the rotation axis. [15] 


\section{Results}

Fig. 4a shows the MFIS of foam I with monomodal pore size distribution for the $1^{\text {st }}$ and $100,000^{\text {th }}$ magneto-mechanical cycles displaying values of 0.10 and $0.115 \%$ indicative of a small in-service training effect [4]. The MFIS after the first four heating/cooling cycles of foam II are shown in Fig. $4 \mathrm{~b}$ together with the MFIS before the first and second heating/cooling cycles. During the first heating/cooling cycle, the MFIS increased from $1.4 \%$ before heating to $2.2 \%$ after cooling to room temperature, where the foam is fully martensitic. At the end of the $1^{\text {st }}$ temperature cycle, the temperature decreased rapidly to $<-100^{\circ} \mathrm{C}$ leading to inter-martensitic transitions [19]. Thus, upon heating back to room temperature, the MFIS was strongly reduced to $0.2 \%$. However, at the end of the second temperature cycle, the MFIS reached 2.5\%, thus exceeding the large strain reached after the first cycle. The MFIS further increased in the $3^{\text {rd }}$ and $4^{\text {th }}$ temperature cycles, achieving an remarkably high value of $8.7 \%$ at the end of the $4^{\text {th }}$ cycle, as shown in Fig. $4 \mathrm{~b}$ (a more complete description of these results is given in [15]).
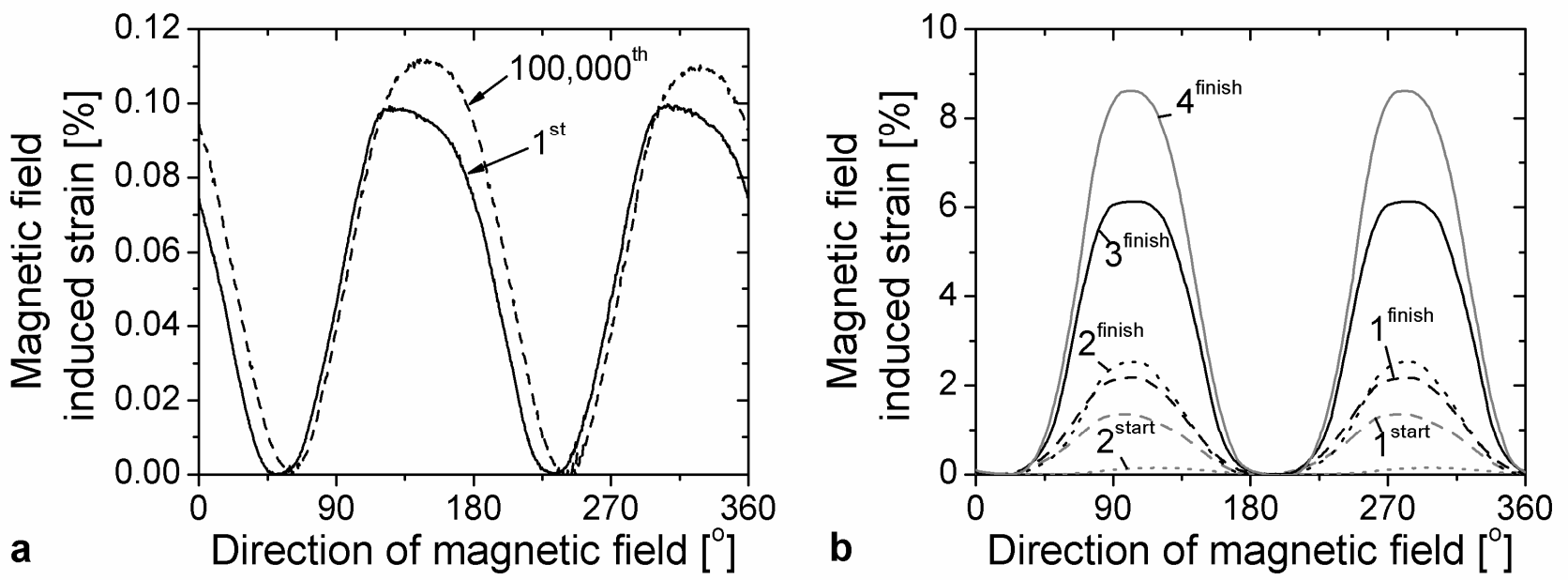

Fig. 4: Plots of MFIS vs. magnetic field orientation for Ni-Mn-Ga foam. (a) Foam I with monomodal pore size distribution displayed MFIS of $0.1 \%$, which slightly increased to $0.115 \%$ during continuously rotating the magnetic field. [14] (b) Foam II with bi-modal pore size distribution showing individual magnetic cycles before and after heating/cooling cycles 1 to 4 (numbers correspond to the thermal cycle, with superscripts "start" and "finish" referring to the strain before austenite start upon heating and after martensite finish upon cooling). A strong training effect results from thermo-magneto-mechanical training. All data were recorded at room temperature. [15]

\section{Discussion}

The results demonstrate that porosity increases the MFIS in polycrystalline Ni-Mn-Ga by reducing the effect of constraints imposed by grain boundaries and thus enabling twin boundary motion. Small and large pores in foam II take on different roles. Large pores naturally relax stresses on long distance, i.e. on the order of the pore size. Foam I, which contains only large pores, displays significant MFIS of $0.1 \%$, which is similar to the best commercial magnetostrictive material, Terfenol-D. While small, this strain is technologically useful and demonstrates that twin boundaries can move in foam I. This strain, however, is much smaller than the MFIS of foam II with bimodal grain size distribution. What then is the role of the small pores?

In some of the large struts of foam I, twins span from one pore to the next, whereas in other struts and in the large nodes, twins obstruct each other (Fig. 2a). These twin structures cannot be completely rearranged with a magnetic field $[20,21]$ especially over grain boundaries in some of the large nodes and wide struts of foam I. In foam II with bimodal pore size distribution, these large 
struts and nodes are subdivided into smaller struts, with twins spanning from one pore to the next (Fig. 2b). Thus, these struts can be compared to single crystals with only one set of active twins. In such twin structures, twin boundary motion can be initiated with a magnetic field $[2,21]$. Thus, when a large strut or node is subdivided by small pores, internal constraints within the large strut or node are relaxed, thereby enabling magnetoplasticity of the entire structure.

Due to different crystallographic orientation, each strut would deform to a different degree if it was not connected via nodes to neighboring struts. For interconnected struts, the strain of neighboring struts must be compatible to some degree. However, neighboring struts may still deform in different ways. Subtle twin boundary tilting allows for bending [22]. Local bending is possible in foam but it is not possible in bulk polycrystalline material. Strut bending permits to transmit strain across unfavorably oriented struts. To what extend strut bending contributes to global strain is subject of further studies.

Finally, pores do not only relax internal constraints. The same ideas can analogously be applied to external constraints such as the coupling to the sample holder, which in this case occurs via stiff glue. The glue makes the sample holder to act as a near-rigid boundary. Thus, the cross contraction/expansion, which accompanies the extension/contraction of the sample in $z$ direction (Fig. 3), is suppressed near the sample holder and the sliding head. In bulk single crystals, this external constraint significantly obstructs twin boundary motion in a large volume fraction thereby reducing MFIS [4]. Furthermore, the interactions of coarse twins, which are the result of external constraints, cause crack nucleation and failure during dynamic experiments with a continuously rotating magnetic field. Strut bending may accommodate deformation locally and act as 'buffer' between the extensively deforming foam and the near-rigid surface layer. No coarse twins can form in thin struts. Thus, the damage mechanism causing fatigue in single crystals does not operate in foam with bimodal pore size distribution. The buffer effect of foam provides a major advantage compared to bulk single crystals as it allows gripping a transducer element in a given sensor and actuator application in any arbitrary way without reducing MFIS. The foam material is furthermore expected to sustain magneto-mechanical cycling by extending the materials fatigue life time. Thus, foams are much more 'design tolerant' than bulk single crystals.

\section{Conclusions}

Magneto-mechanical experiments with Ni-Mn-Ga foam with monomodal and bimodal pore size distribution show that

- MFIS comparable to those of bulk single crystals can be achieved in cast, porous, polycrystalline MSMA.

- Small pores increase the fraction of struts with twins spanning from one pore to the next, thereby creating single-crystal-like MFIS in these struts and facilitating magnetic-fieldinduced twin boundary motion throughout the foam.

- Small pores effectively reduce internal and external constraints, thereby providing a mechanism to grip MSMA transducers in real applications, and may thus extend the lifetime of Ni-Mn-Ga transducers under cyclic loading conditions.

\section{Acknowledgements}

PM thanks the organizers of ICFSMA'09 for the invitation to give an invited talk and for supporting his participation. The authors thank Adrian Rothenbühler (Boise State University) for assistance with magnetic and magneto-mechanical experiments. This project was funded by the National Science Foundation through grant NSF-DMR 0804984 (Boise State University) and DMR-805064 (Northwestern University). MC acknowledges partial financial support through the German Research Foundation (DFG) priority program SPP 1239 (grant No. Schn 1106/1). PM is thankful to ETH Zürich for donating magneto-mechanical testing devices. 


\section{References}

[1] A. Sozinov, A. A. Likhachev, N. Lanska, K. Ullakko: Applied Physics Letters Vol. 80 (2002), p. 1746

[2] S. J. Murray, M. Marioni, S. M. Allen, R. C. O'Handley, T. A. Lograsso: Applied Physics Letters Vol. 77 (2000), p. 886

[3] P. Müllner, V. A. Chernenko, G. Kostorz: Journal of Applied Physics Vol. 95 (2004), p. 1531

[4] M. Chmielus, V. A. Chernenko, W. B. Knowlton, G. Kostorz, P. Müllner: European Physical Journal-Special Topics Vol. 158 (2008), p. 79

[5] M. Marioni, D. Bono, A. B. Banful, M. del Rosario, E. Rodriguez, B. Peterson, S. M. Allen, R. C. O'Handley: Journal De Physique IV Vol. 112 (2003), p. 1001

[6] P. Lázpita, G. Rojo, J. Gutiérrez, J. M. Barandiarán, R. C. O'Handley: Sensor Letters Vol. 5 (2007), p. 65

[7] U. Gaitzsch, M. Pötschke, S. Roth, B. Rellinghaus, L. Schultz: Scripta Materialia Vol. 57 (2007), p. 493

[8] U. Gaitzsch, M. Pötschke, S. Roth, B. Rellinghaus, L. Schultz: Acta Materialia Vol. 57 (2009), p. 365

[9] U. Gaitzsch, R. Techapiesancharoenkij, M. Pötschke, S. Roth, L. Schultz: IEEE Transactions on Magnetics Vol. 45 (2009), p. 1919

[10] J. Tellinen, I. Suorsa, A. Jaaskelainen, I. Aaltio, K. Ullakko, Basic properties of magnetic shape memory actuators, in: Actuator 2002, Wirtschaftsfoerderung Bremen GmbH, Bremen, 2002, pp. 566-569

[11]P. Müllner, A. V. Chernenko, D. Mukherji, G. Kostorz, Cyclic magnetic-field-induced deformation and magneto-mechanical fatigue of Ni-Mn-Ga ferromagnetic martensite, in: Y. Furuya, E. Quandt, Q. Zhang, K. Inoue, M. Shahinpoor (Eds.), MRS Fall Meeting 2003, Vol. 785, MRS, Boston, MA, 2003, pp. 415-420

[12] O. Heczko, L. Straka, O. Söderberg, S. P. Hannula, Magnetic shape memory fatigue, in: W. D. Armstrong (Ed.) Smart Structures and Materials 2005, San Diego, CA, 2005, SPIE Proc. Vol 5761, pp. 513-520

[13]P. Müllner: Materials Science and Engineering A Vol. 234 (1997), p. 94

[14] Y. Boonyongmaneerat, M. Chmielus, D. C. Dunand, P. Müllner: Physical Review Letters Vol. 99 (2007), p. 247201

[15] M. Chmielus, X. X. Zhang, C. Witherspoon, D. C. Dunand, P. Müllner: Nature Materials (2009), published electronically on September 13. DOI:10.1038/NMAT2527. In press.

[16] Y. Boonyongmaneerat, D. C. Dunand: Advanced Engineering Materials Vol. 10 (2008), p. 379

[17]I. Aaltio, K. Ullakko, Magnetic shape memory (MSM) actuators, in: Actuator 2000, Wirtschaftsfoerderung Bremen GmbH, Bremen, 2000, pp. 527-530

[18]P. Müllner, V. A. Chernenko, M. Wollgarten, G. Kostorz: Journal of Applied Physics Vol. 92 (2002), p. 6708

[19] C. Segui, V. A. Chernenko, J. Pons, E. Cesari, V. Khovallo, T. Takagi: Acta Materialia Vol. 53 (2005), p. 111

[20] K. Ullakko, J. K. Huang, C. Kantner, R. C. OHandley, V. V. Kokorin: Applied Physics Letters Vol. 69 (1996), p. 1966

[21]P. Müllner, G. Kostorz: Materials Science Forum Vol. 583 (2008), p. 43

[22]H. D. Chopra, C. Bailly, M. Wuttig: Acta Materialia Vol. 44 (1996), p. 747 